\title{
A Review of Algorithms for Order Batching Problem in Distribution Center
}

\author{
Tingwei Ma \\ Automation School. Beijing University of Post and \\ Telecommunications, BUPT \\ Beijing, China \\ e-mail: mtw1988@bupt.edu.cn
}

\author{
Peng Zhao \\ Automation School. Beijing University of Post and \\ Telecommunications, BUPT \\ Beijing, China \\ e-mail: zhaopengali@126.com
}

\begin{abstract}
Order batching is pivotal for the efficiency of order picking operations so that researches on this problem are prevalent. The aim of this paper is to analyze and summarize the range and idea of order batching by giving an review of the different solution approaches which have been suggested in the literature, and at last indicate tendency of future research of order batching.
\end{abstract}

Keywords-order batching; manual picking; algorithms; time window; performance

\section{INTRODUCTION}

Recent tendency shows that the demand of customer has been changing from large amount and small variety orders to small amount and large variety orders, and no matter when orders arrive they should be picked and delivered correctly and timely. This requires companies and enterprises who want to gain advantages to establish more efficient and flexible order picking system in competitive market. Order picking is the most important activity in distribution center and its costs may account for $50 \%-65 \%$ of overall operating costs for warehouse. The efficiency of this activity will directly affect customer service level. Despite several attempts to make this procedure become automatic order picking still requires a lot of manual work in practice, so that most literatures concentrate on manual picking system. Manual picking system can be distinguished into two kinds: picker-to-parts system and parts-to-picker system. With respect to system of first kind, managers face three planning problems on operative level, they are storage location, order batching and picker routing problem. Among these problems order batching is proofed to be critical as good batch method contributes greatly to the productivity as well as flexibility via reducing the time needed to process customer orders.

\section{OVERVIEW OF ALGORITHMS IN ORDER BATCHING PROBLEM}

Order batching problem is known to be NP-hard problem. Though some of researches are based on optimization approaches, the results are limited in small scale situation. Thus using heuristic algorithm to deal with order batching is almost become common sense.

\section{A. Classical Algorithms}

The classical algorithms include priority rule-based algorithm, seed algorithm and saving algorithm. In priority rule-based algorithm, customer orders are assigned successively to batches in accordance with priorities assigned previously. First-Come-First-Server (FCFS) rule probably the most straightforward one where priority is assigned as customer order come in. Seed algorithm is introduced by Elsayed [1] in 1981, it generate batches sequentially in two phases. An initial order (seed) is chosen for a batch in selection phase, afterwards unsigned customer orders are added to the seed according to an order-congruency rule in matching phase. The methods related to selection of seed order and order-congruency rule is considered by To \& Tseng et al. [2, 3]. Saving algorithm is based on the algorithm introduced by Clarke and Wright [4] in 1964 which has been applied in several ways for order batching problem. Savings are computed which can be obtained in terms of tour length reduction by assigning the items of customer orders to one batch instead of collecting them separately. In the improved version of Elsayed and Unal [5] which is known as C\&W(ii) savings are recalculated each time a new assignment of customer orders to batches has been made. Classical algorithms illustrate general methods for the study of batching issue and indicate a path for subsequent researchers. These algorithms are still active in recent studies.

\section{B. Data Mining Approach}

Hwang et al. [6] consider order batching problem as a clustering problem, they bring two feature vectors to determine picking locations for each order. Three similarity measures are defined correspondingly. Their study offers a new sight of this the problem. Lee and Du [7] introduce three formulas to calculate similarity factor based on cluster analysis, they also establish cluster model for Order batching problem in which batching results are calculated through a heuristic algorithm similar to saving algorithm. After that routing length of picker is taken into account according to the batching result. For in the whole batching procedure walking distance is not involved so that the algorithm is highly simplified. Chen et al. [8] bring Association Rule (AS) into order batching. It sets foundation for the method raised by $\mathrm{Chen}$ and $\mathrm{Wu}$ [9] which combines data mining and integer programing. In this method, at first similarities of customer orders are determined by means of an association rule. For each pair of orders an order correlation measure is obtained. 0-1 integer programing approach is then used to cluster customer orders into batches. Hsieh and Huang [10] develop two new heuristics called K-means batching 
(KMB) and Self-organization Map Batching (SOMB) using data mining approach. These heuristics are discussed under four different order types respectively and compared with AS Particle Swarm Optimization Batching Method (PSOBM) in terms of routing distances CPU calculation time as well as utilization of picking devices.

\section{Local Search}

The general principle of Local Search (LS) heuristics consists of exploring the neighborhood of a solution in order to identify a new solution with smaller objective function value. Gademann and Van de Velde [11] first bring LS to the order batching problem. They introduce a method which uses FCFS rule to generate initial solution and exchanges two orders come from different batches to generate neighborhood. Henn et al. [12] develop Iterated Local Search (ILS) which adds perturbation phase into LS. ILS tries to intensify the search for improved solutions in the vicinity of local minima. Perturbation phase is realized through moving a number of orders that randomly generated from one batch to another. Albareda et al. [13] apply Variable Neighborhood Search (VNS) to the order batching problem. They define three different kinds of neighborhoods based on the following moves: (1) Assignment of one order to a different batch. (2) Assignment of two orders from one batch to other batches. (3) Assignment of two orders of one or two batches to one or two other batches. The algorithms terminates if the incumbent solution is optimal for all three neighborhoods.

\section{Tabu Search}

Tabu Search (TS) developed by Glover in 1986 aims at simulating human memory processes. The algorithm tries to avoid cycling search by a so-called tabu list. Three configurations should be taken into account for a certain TS application. The first is the method which can be used to generate initial solution, the second indicates the neighborhood structure which grouping solutions through one step transform from current solution, and the third defines the way in which the neighborhood is explored. In the article of Henn and Wäscher [14] two solutions are adopted namely TS and Attribute-Based Hill Climber (ABHC) which introduced in [15]. The ABHC characterizes the corresponding solutions as a set of attributes which could be any solution feature. Similar to TS the algorithm starts with an initial solution and search for a new incumbent solution in each iteration, however only neighbors of the incumbent solution are considered which containing at least one attribute. Kulak et al. [16] improve the TS, they propose two new Tabu Search based on cluster analysis applied in multi-aisles warehouse system to solve order batching and picker routing problem.

\section{E. Population-Based Algorithms}

The Rank-Based Ant System (RBAS) is a populationbased solution approach in which each ant presents a single solution. Henn et al. [12] modify RBAS for order batching problem. In their method an order is assigned to a batch at first, next calculate savings and strength of pheromone for each combination of two orders. Savings and pheromone intensity define the possibility of combination. LS function is applied for improving solution if no further feasible combinations of batches can be identified.
Another population-based algorithm which is prevalent in dealing with order batching problem is Genetic Algorithm (GA) introduced by Holland in 1975. GA iteratively generates a large number of possible solutions. Each of the solution is defined as an individual that could transfer their genes to next generations through mutation and crossover. This means the solution with better gene has more probability to survive. Hsu et al. [17] propose a solution to order batching problem with GA which each solution is represented by a string of integers which groups each customer order into a particular batch. The fitness of a solution is calculated by the length of tour between the longest in population and current solution. Koch and Wäscher [18] develop a mixed algorithm with GA and LS the result shows this method predominate the benchmark algorithm in Hsu's. Tsai et al. [19] come up with a batching model which considers both travel distance and due date. GA is used to solve the order batching and picker routing problem at the same time. Based on the study of Hsu, Wan et al. [20] consider the effect of seed order selection on optimization result. Wang [21] develop multiobjective model of order batching in order picking procedure, which considers picking rout, picking times and lead time. They solve the model with FCFS, GA and Improved Niche Genetic Algorithm (INGA) respectively.

\section{F. Time Window Approach}

Unlike the static order batching problem, a more real case is that orders arrive randomly during the time of processing previous orders. And before new order arrives the information is not available. The study under this circumstance calls dynamic order batching or on-line batching. In dynamic batching time window approach is widely used which can be carried out in two different ways, namely variable time window and fixed time window. In variable time window batching it is usually assumed that the capacity of the picking device is defined in the number of customer orders, and pickers proceed after certain amount of orders arrive. In fixed time window batching all customers orders arriving during a particular time interval are assigned to batches. Chew and Tang [22] present a travel time model of a single block order picking warehouse with variable time window batching. They design a queuing network with two queues. In the first one, customer orders arrive according to a Poisson-process and batches are generated by means of the FCFS rule. If a particular number of customer orders is in the first queue, these orders are assigned to a batch and are moved to the second queue. Le-Duc et al. [23] carry out a similar investigation of random customer order for a two block layout warehouse. A corresponding model of fixed time window is presented by Van Nieuwenhuyse and De Koster [24]. Henn [25] tries to modify method in static order batching to dynamic order batching. In his model orders from customers arrive at different time point and three types of decision point are defined. At each decision point, orders which have not been assigned are considered as static. That means dynamic batching is divided into many static batching problem. For every batch decision is made whether start immediately or postpone for future possible combination. Ma and Wen [26] bring the idea of postponed manufacturing to picking process in warehouse, and propose a dynamic time window strategy based on 
time delay. The strategy can eliminate the phenomenon of waiting time and block demand that exists in the current picking system and greatly enhance the efficiency of picking system.

\section{G. Other approaches}

Matusiak et al. [27] inspired by a large retail warehouse in Finland, they manager to combine two different strategies. A fast simulated annealing algorithm is applied in solving order batching problem and $\mathrm{A}^{*}$-type shortest path algorithm used for calculating travel distance. The global objective is to minimize travel distance. The numerical experiment shows that this approach saves nearly $16 \%$ of total distance when compared to benchmark. Gademann et al. [28] study wave picking model which a large set of customer orders (wave) for a joint destination is released simultaneously. They adopt branch-and-bound method to obtain the solution which aims at minimizing lead time for each order. Bukchin et al. [31] according to Markov Decision Process (MDP) propose an algorithm called MDP-H for dynamic order batching problem. Lee [32] proposes the envelop algorithm for order batching operation on the basis of the bin packing algorithm considering the location of items in warehouse.

\section{PERFORMANCE OF HEURISTICS}

An up-to-date study which considers a comprehensive set of problem parameters and provides an in-depth analysis of performance of a representative set of the solution methods for the order batching problem does not exist in the literature so far. Since very diverse settings and circumstances, arguments of performances among heuristics are limited in a narrow field.

Ho and Tseng et al. analyze the performance of seed algorithms. They compare various combinations of seed selection and order congruency rules to each other. In [2] a combination of Smallest Number of Picking Aisles (seed selection rule) and Smallest Number of Additional Picking Aisles (order-congruency rule) gives the smallest tour lengths. In [3] the result can be improved through combining Smallest Number of Picking Aisles and Shortest Average Mutual-Nearest-Aisle Distance. In [12] the authors compare their versions of ILS and RBAS against FCFS, C\&W(ii) and LS. By means of S-shape and Largest Gap routing strategies, they find ILS and RBAS reduce the total tour length approximately by $20 \%$ in comparison to $17 \%$ that of FCFS-C\&W(ii). Albareda et al. [13] benchmark VNS against FCFS, and several seed and savings approaches. Among these heuristics VNS is able to find the best solution in most cases and improves the results obtained by FCFS by $19 \%$ on average. In [14] C\&W(ii) and LS are combined as benchmark to evaluate the performance of TS and ABHC heuristics with S-shape and Largest-Gap routing strategies. ILS is also taken into account. The result of numerical experiment shows that TS and $\mathrm{ABHC}$ are superior to existing algorithms.

As concerned above, it is difficult to compare the presented result with each other due to the different parameter settings used in numerical experiments. To make results more comparable, it would be useful for future research to design experiment similar to existing ones.

\section{CONCLUSIONS AND FUTURE RESEARCH DIRECTIONS}

\section{A. Conclusions}

This paper reviews the order batching problem at the angle of heuristics. Some of researchers attempt to apply heuristics which have been successfully adopted in other areas including data mining, neural network, artificial intelligence and so on. However, the published result of numerical experiment is based on distinct configurations such as dimension and size of the warehouse, quantity and scale of customer orders, capacity of picking devices, selection of routing strategies and so on. As a result, it seems impossible to draw a general conclusion like whether one heuristic is better than another. In spite of this, exploring faster algorithm is usually worth-while. When retrospect the history of order batching problem, focus has changed from desire to promote productivity and control the costs to accelerate respond and raise customer service level. This change react the fact that new requirements are continuously arising for any entities in supply chain. For order batching problem, it makes researchers consider more about the circumstances in real world. Like considering due date batching problem, dynamic batching and so on, future research may offer more details in literatures.

\section{B. Future research directions}

Although it is difficult to develop a universal model as concerned with diversities in real warehouses, a comprehensive in-depth study on application of algorithms with different warehouse layouts is aspired. Furthermore, indicators in regard to customer service level should be more included in existing models where complexity of algorithm is acceptable. Future research could concentrate on the following aspects: (1) Develop algorithms including mixed algorithms to solve large scale problem; (2) Transplant algorithms with different configurations in warehouse; (3) Go deep into dynamic order batching problem; (4) Study interaction of order batching with the other related strategies.

\section{ACKNOWLEDGMENT}

The research has been supported by Industrial Project Foundation of Guizhou province, China, grant GY [2013] 3012 .

\section{REFERENCES}

[1] E. A. Elsayed, "Algorithms for optimal material handling in automatic warehousing systems,',International Journal of Production Research, vol.19, pp:525-535, 1981

[2] Y.-C. Ho, Y.-Y Tseng, "A study on order-batching methods of order-picking in a distribution center with two cross aisles," International Journal of Production Research,vol.44,pp: 3391-3417, 2006

[3] Y.-C. Ho, T.-S. Su, Z.-B. Shi, "Order-batching methods for an order-picking warehouse with two cross aisles,"Computers\& Industrial Engineering, vol. 55, pp:321-347, 2008

[4] G. Clarke, J.W Wright, "Scheduling of Vehicles from a Central Depot to a Number of Delivery Points," vol.12, pp:568-581, 1964

[5] E. A. Elsayed, O. I. Unal, "Order batching algorithms and traveltime estimation for automated storage/retrieval systems," International Journal of Production Research,vol.27, pp:1097-1114, 1989 
[6] H. Hwang, W. Beak, M. Lee, "Cluster algorithms for order picking in an automated storage and retrieval system," International Journal of Production Research, vol.26, pp: 189-201, 1988

[7] S.-Z. Lee, W.-H. Du, "Order batching model and heuristic based on cluster analysis," Statistics and Decision-making, vol.12, pp:5356,2008 (in Chinese)

[8] M.-C. Chen, C.-L Huang, K.-Y. Chen, H.-P. Wu, "Aggregation of orders in distribution centers using data mining," Expert Systems with Applications, vol.28, pp:453-460, 2005

[9] M.-C. Chen, H.-P. Wu, “An association-based clustering approach to order batching considering customer demand pattern," Omega, vol.33, pp:333-343, 2005

[10] L.-F. Hsieh, Y.-C Huang, "New batch construction heuristics to optimise the performance of order picking systems," International Journal of Production Economics, vol.13, pp: 618-630, 2011

[11] N. Gademann, S. Van de Velde, "Order batching to minimize total travel time in a parallel-aisle warehouse," IIE Transactions, vol.37, pp:63-75, 2005

[12] S Henn, S Koch, K.F. Doerner, "Metaheuristics for the Order Batching Problem in Manual Order Picking Systems," BuRBusiness Research, vol.3, pp:82-105, 2010

[13] M Albareda-Sambola, A Alonso-Ayuso, E Molina, CS. de Blas, "Variable neighborhood search for order batching in a warehouse," Asia-Pacific Journal of Operational Research, vol.26, pp:655-683, 2009

[14] S Henn, G Wäscher, "Tabu search heuristics for the order batching problem in manual order picking systems," European Journal of Operational Research, vol.222, pp: 484-494, 2012

[15] M Whittley lan, D Smith Georgel, "The Attribute Based Hill Climber," Journal of Mathematical Modelling and Algorithms, vol.3, pp:167-178, 2004

[16] O Kulak, Y Sahin, M.E. Taner, "Joint order batching and picker routing in single and multiple-cross-aisle warehouses using clusterbased tabu search algorithms,'Flexible Services and Manufacturing Journal,vol.24, pp:52-80, 2012

[17] C.-M. Hsu, K.-Y. Chen, "Chen M.-C. Batching orders in warehouses by minimizing travel distance with genetic algorithms," Computers in Industry,vol. 56, pp:169-178, 2005

[18] S Koch, G. A. Wäscher, "Grouping Genetic Algorithm for the Order Batching Problem in Distribution Warehouses," German:Otto-von-Guericke-Universitat Magdeburg, 2011
[19] C.-Y. Tsai, Liou, T.-M. Huang, "Using a multiple-GA method to solve the batch picking problem: considering travel distance and order due time," Interational Journal of Production Research, vol.46,pp:6533-6555, 2008

[20] J. Wan, S.-Q. Zhang, L Lee, "Research on Distance Optimazion of Batch-order Picking Based on GA ," Journal of HeBei University of Technology,vol.38, pp:10-14, 2009 (in Chinese)

[21] Y.-Y. Wang, Y.-H Wu, "Research on strategy of order batching in distribution center"Journal of ShanDong University (engineering version),vol.2, pp:43-46, 2010 (in Chinese)

[22] E Chew, L Tang, "Travel time analysis for general item location assignment in a rectangular warehouse," European Journal of Operational Research,vol.112, pp:582-597,1999

[23] T Le-Duc, R De Koster, "Travel time estimation and order batching in a 2-block warehouse," European Journal of Operational Research,vol.176, pp:374-388,2007

[24] I Van Nieuwenhuyse, R De Koster, "Evaluating order throughput time in 2-block warehouses with time window batching,' International Journal of Production Economics,vol.121, pp:654 664, 2009

[25] S Henn, "Algorithms for on-line order batching in an order picking warehouse," Computer \& Operations Research,vol.39, pp:25492563, 2012

[26] S.-H. Ma, J. Wen, “The Order Batching Strategies Based on Time Postponement," Industrial Engineering and Management, 2004 (in Chinese)

[27] M Matusiak, R De Koster, Leo K, Saarinen J, "A fast simulated annealing method for batching precedence constrained customer orders in a warehouse," European Journal of Operational Research, 2013(Article in press)

[28] N. Gademann, Van de Berg, Van Der Hoff, "An order batching algorithm for wave picking in a parallel aisle warehouse," IIE Transactions, vol.33, pp:385-398, 2001

[29] Y Bukchin, E Khmelnitsky, P Yakuel, "Optimizing a dynamic order-picking process," European Journal of Operational Research, vol.219, pp:335-346, 2012

[30] S.-Z. Lee, "Batch order picking model and envelop algorithm in distribution center," Lifting and Transforming Machines, pp:19-22, 2009 (in Chinese) 